\section{Author's Reply \\ The Dose-Dependent Relationship Between CRP and MS With the Japanese Definition}

We appreciate the letter by Dr Oda regarding our recent paper "Metabolic syndrome and C-reactive protein in the general population: the JMS Cohort Study"! As pointed out the cut-off points of waist circumference (WC) were decided by arbitrary and inconsistent methods and new criteria of metabolic syndrome (MS) in which WC is replaced by Creactive protein (CRP) have been proposed? Also, it was highlighted that it remains controversial whether or not the WC component in the Japanese definition and diagnostic criteria of MS is rational. However, once the definition and criteria were made, we think it was reasonable to use those criteria and if the diagnostic criteria were incomplete, evidence should be compiled from various studies to modify them.

Some studies were examined MS and CRP in Japan using the criteria of the National Cholesterol Eduration Program (NCEP), or NCEP with modification:-5 Oda et al used body mass index $\geq 25 \mathrm{~kg} / \mathrm{m}^{2}$ as obesity, instead of WC, in their study of the cut-off points of CRP because they did not have data for the WC of the subjects at heath check-ups at hospital? Our study was population-based, as was the Hisayama study, and the subjects were rather healthier as we previously reported? That is why the CRP values were lower than those in other Japanese studies. Moreover, the data were obtained in the first half of the 1990s. We used the Japanese criteria in our study, and the proportion of MS was much lower in women than in men $(1.8 \%$ vs $9.4 \%)$. That large discrepancy was mainly related to the WC component in the Japanese criteria of MS. Our study had some study limitations as did the others.

We calculated the proportion of MS and the odds ratios (OR) of MS by CRP tertiles using WC $\geq 90 \mathrm{~cm}$ in men and $\mathrm{WC} \geq 80 \mathrm{~cm}$ in women. The proportion of MS was $4.7 \%$ in men and $7.5 \%$ in women, and the OR was $3.2(95 \%$ confidence interval: 1.8-5.7) and 5.6 (3.2-9.9) instead of 2.9 (1.5-5.9) and $5.7(31.0-11.1)$, and the data were identical. Our study did not examine if the Japanese criteria of MS were rational, but examined only the relationship between MS and CRP using the extant Japanese criteria. Therefore, we could not verify the validity of those criteria. We appreciate our data would become part of the evidence for future changes.

\section{References}

1. Ishikawa S, Kayaba K, Gotoh T, Nakamura Y, Kajii E. Metabolic syndrome and C-reactive protein in the general population. Circ J 2007; 71: 26-31.

2. Oda E, Oohara K, Abe A, Veeraveedu PT, Watanabe K, Kato K, et al The optimal cut-off point of C-reactive protein as an optional component of metabolic syndrome in Japan. Circ J 2006; 70: 384-388.

3. Tamakoshi K, Yatsuya H, Kondo T, Hori Y, Ishikawa M, Zhang H, et al. The metabolic syndrome is associated with elevated circulating $\mathrm{C}$-reactive protein in healthy reference range, a systemic low-grade inflammatory state. Int J Obes Relat Metab Disord 2003; 27: $443-$ 449.

4. Nakanishi N, Shiraishi T, Wada M. C-reactive protein concentration is more strongly related to metabolic syndrome in women than in men: The Minoh Study. Circ J 2005; 69: 386-391.

5. Takeuchi H, Saitoh S, Takagi S, Ohnishi H, Ohhata J, Isobe T, et al. Metabolic syndrome and cardiac disease in Japanese men: Applicability of the concept of metabolic syndrome defined by the National Cholesterol Education Program-Adult Treatment Panel III to Japanese men: The Tanno and Sobetsu Study. Hypertens Res 2005; 28: 203-208.
6. Doi Y, Kiyohara Y, Kubo M, Ninomiya T, Wakugawa Y, Yonemoto $\mathrm{K}$, et al. Elevated C-reactive protein is a predictor of the development of diabetes in a general Japanese population: The Hisayama Study. Diabetes Care 2005; 28: 2497-2500.

7. Ishikawa S, Gotoh T, Nago N, Kayaba K. The Jichi Medical School (JMS) Cohort Study: Design, baseline data and standardized mortality ratios. J Epidemiol 2002; 12: 408-417.

Shizukiyo Ishikawa (on behalf of the authors) Division of Community and Family Medicine Center for Community Medicine, Jichi Medical University, Shimotsuke, Japan 